\title{
Stereoselective Synthesis of Carbohydrate-Based Fused Bicyclic $\delta$-Lactones
}

\author{
Renata B. de Oliveira ${ }^{a}$, Ricardo J. Alves ${ }^{*, b}$, José Dias de Souza Filho ${ }^{a}$ and \\ Maria Auxiliadora F. Prado ${ }^{b}$ \\ ${ }^{a}$ Departamento de Química, Instituto de Ciências Exatas, Universidade Federal de Minas Gerais, \\ Av. Antônio Carlos 6627, 31270-200 Belo Horizonte - MG, Brazil \\ ${ }^{b}$ Departamento de Produtos Farmacêuticos, Faculdade de Farmácia, Universidade Federal de Minas Gerais, \\ Av. Olegário Maciel 2360, 30180-112 Belo Horizonte - MG, Brazil
}

\begin{abstract}
Relata-se no presente trabalho a síntese de $\delta$-lactonas quirais derivadas de carboidratos, por meio de reações de ciclização radicalar regio- e estereosseletivas. A configuração do novo centro estereogênico depende da estrutura do carboidrato.
\end{abstract}

We describe in the present work the synthesis of carbohydrate-based chiral $\delta$-lactones by regioand stereoselective radical cyclization reactions. The configuration of the newly stereogenic center was found to depend on the structure of the carbohydrate moiety.

Keywords: radical cyclization, carbohydrate and $\delta$-lactones

\section{Introduction}

Free radical cyclization has been applied extensively in natural product synthesis. ${ }^{1}$ A number of biologically active natural products especially pheromones are lactones. ${ }^{2}$ In addition, lactones have assumed importance as building blocks for the synthesis of natural products such as alkaloids, macrocyclic antibiotics and terpenoids. Because of the presence of often numerous chiral centers in natural products, diastereoselectivity is crucial in their synthesis. ${ }^{3}$

Carbohydrate have been used as "off-templates" for the stereoselective synthesis of chiral $\gamma$ - and $\delta$-lactones. ${ }^{4-6}$ In connection with our interest in free radical carbocyclization reactions from carbohydrates, ${ }^{7-9}$ we report herein our results on the regio- and stereoselective synthesis of chiral $\delta$-lactones by 6-exo-trig radical carbocyclization reactions on carbohydrate templates.

The iodo esters 1 to 4 were chosen as radical precursors. They are easily prepared in a few steps using classical carbohydrate chemistry and should allow the comparison of the influence of a pyranose versus a furanose template in the outcome of the radical carbocyclization reaction. The cinnamoyl and crotonyl moieties generate a new chiral

\footnotetext{
* e-mail: ricky@dedalus.lcc.ufmg.br
}

center upon cyclization. Hence, the stereoselectivity of the reaction can be evaluated.

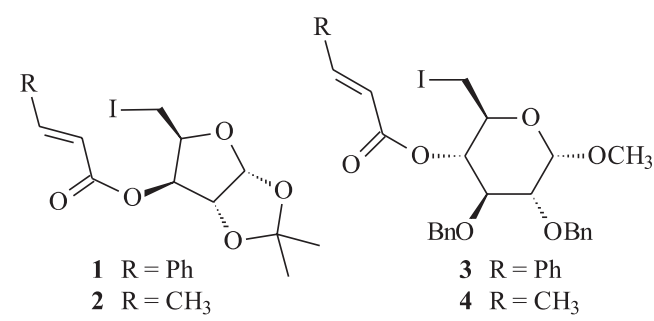

\section{Results and Discussion}

Starting with known 1,2-O-isopropylidene-a-Dxylofuranose $(5)^{10}$ and methyl 2,3-di- $O$-benzyl- $\alpha$-Dglucopyranoside $(6),{ }^{11}$ the radical precursors 1 to $\mathbf{4}$ were prepared in two steps namely, regioselective replacement of the primary hydroxyl group by iodide, ${ }^{12}$ followed by esterification of the remaining hydroxyl group ${ }^{13}$ (Scheme).

The radical carbocyclization reactions of compounds 1 to 4 were then investigated. Thus, a solution of tri- $n$ butyltin hydride (1.2 equivalents) and catalytic amount of azoisobutyronitrile (AIBN) in nitrogen-saturated anhydrous benzene was added slowly (over three hours) to a solution of the radical precursors $\mathbf{1}$ to $\mathbf{4}$ in boiling benzene $\left(0.013 \mathrm{~mol} \mathrm{~L}^{-1}\right)$. 


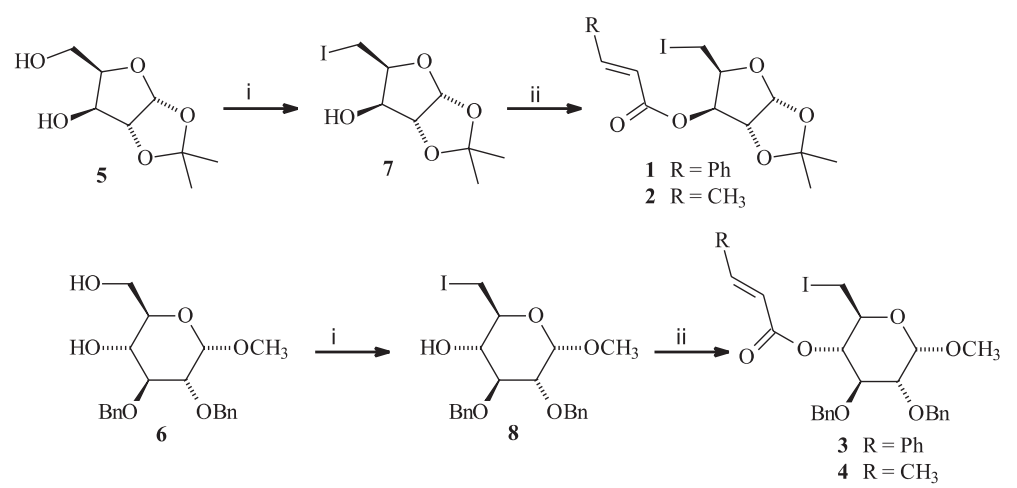

Scheme. Reagents and conditions: i) $\mathrm{I}_{2}, \mathrm{Ph}_{3} \mathrm{P}$, imidazole, toluene, reflux [70\% (7) and 63\% (8)]; ii) cinnamic acid or crotonic acid, DCC, DMAP, $\mathrm{CH}_{2} \mathrm{Cl}_{2}$, rt $(56-79 \%)$.

In all cases, products of completely stereoselective 6exo-trig carbocyclization were formed in 1-30\% yield by addition of the alkyl radical to the $\alpha$-position of $\alpha, \beta$ unsaturated esters. The corresponding hydrogenolysis products were also isolated in 42-70 \% yield (Figure 1).

The results are summarised in the Table 1.

Several authors reported failure in attempting 5- and 6exo-trig radical ring closure of ester derivatives due to the geometric constraints associated with the preferred s- $Z$ conformation of the ester function. ${ }^{14-20}$ Thus, the successful
Table 1. Cyclization and reduction products from radical precursors 1 to 4

\begin{tabular}{|c|c|c|c|}
\hline \multirow{2}{*}{$\begin{array}{l}\text { Radical } \\
\text { Precursor }\end{array}$} & \multicolumn{2}{|c|}{ Product $(\text { yield } \%)^{\mathrm{a}}$} & \multirow{2}{*}{$\begin{array}{l}\text { Stereochemistry } \\
\text { at } \alpha-C^{b}\end{array}$} \\
\hline & $\delta$-lactone & reduction & \\
\hline 1 & $9(30)$ & $13(42)$ & $S$ \\
\hline 2 & $10(15)$ & $14(66)$ & $S$ \\
\hline 3 & $11(22)$ & $15(53)$ & $R$ \\
\hline 4 & 12 (1) & $16(70)$ & $R$ \\
\hline
\end{tabular}

${ }^{\mathrm{a}}$ Yields after purification by column chromatography; ${ }^{\mathrm{b}}$ Deduced by NOESY experiments.

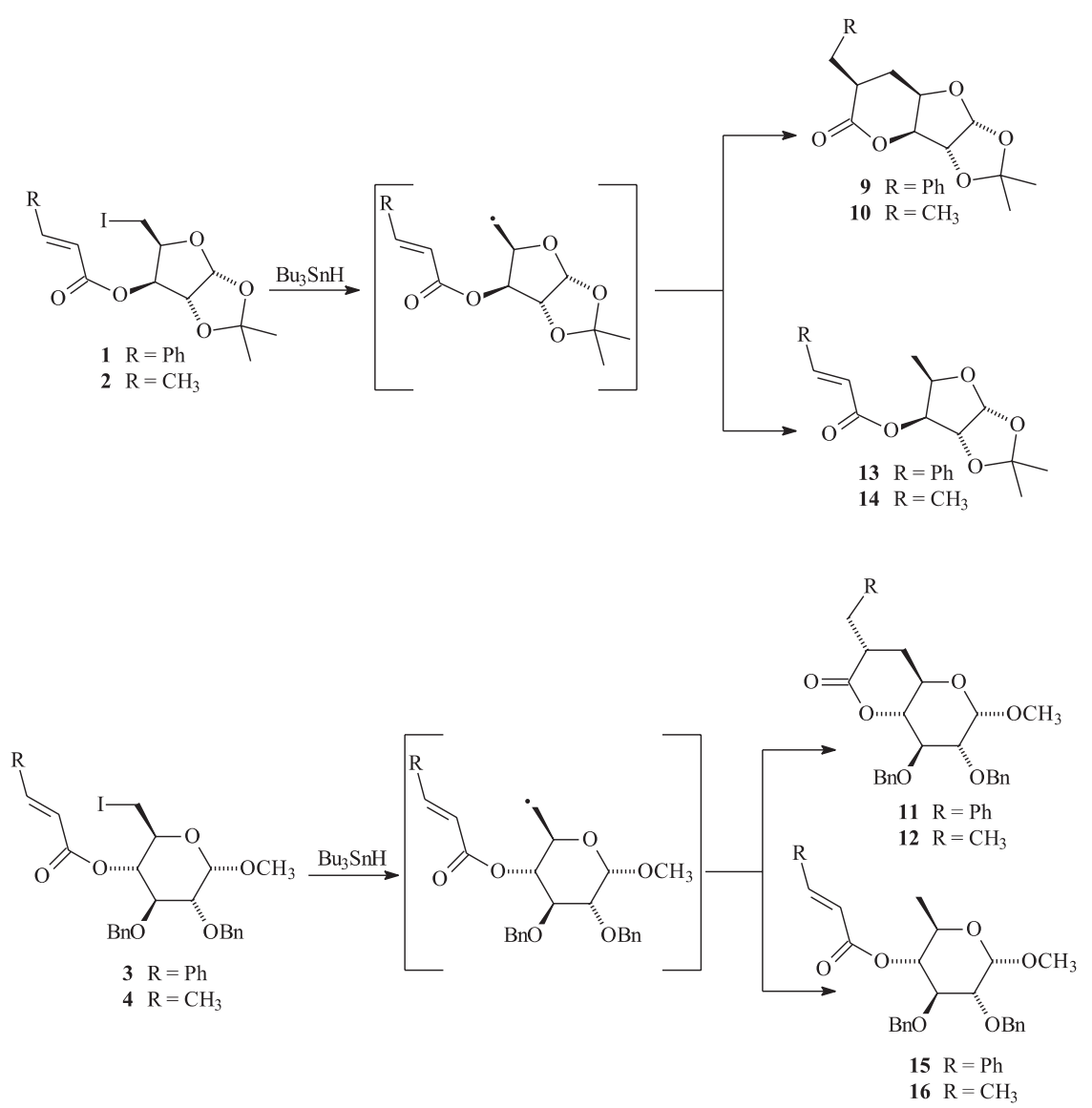

Figure 1. Products of the reactions of precursors 1 to 4 with $\mathrm{Bu}_{3} \mathrm{SnH}$. 
outcome of the reactions in our study, despite the low yields of the cyclized products, may be related to the conformational restraints imposed by the carbohydrate moiety. As shown in the Table 1, the cinnamoyl precursors (1 and $\mathbf{3}$ ) gave better yields of the corresponding $\delta$-lactones. This was attributed to the stability of the intermediate benzyl radical. According to the literature ${ }^{21}$ the formation of fused rings in radical ring closure is predominately or exclusively cis for small rings (six or fewer atoms). Thus, the cis-relationship between the substituents at C-3 and C-5 of the radical esters in the furanose ring form may account for the better yields of cyclized products in this series.

The regioselectiviy of the reaction was deduced by analysis of the NMR spectra. In the case of the crotonyl radical precursors 2 and 4, the 6-exo-trig cyclization mode was indicated by the presence of the ethyl group signals in the ${ }^{1} \mathrm{H}$ NMR spectra of the respective products $\mathbf{1 0}$ and $\mathbf{1 2}$.

For $\delta$-lactones 9 and 11 the analysis of the ${ }^{1} \mathrm{H}$ NMR spectra was not conclusive. Thus, the connectivity was confirmed by analysis of HMBC contour plots, which showed coupling between H-5, H-5' (lactone 9) and H-6, H-6' (lactone 11) and the carbonyl carbon (Figure 2).

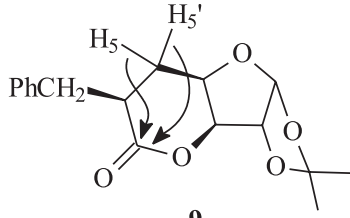

9

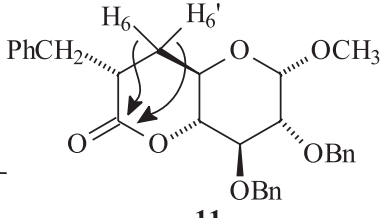

11
$\mathrm{HMBC}:{ }^{3} J_{\mathrm{C}, \mathrm{H}}$

Figure 2. Three-bond correlation between H-5, H-5' (lactone 9) or H-6, H-6' (lactone 11) and the carbonyl carbon observed by analysis of the HMBC contour maps.
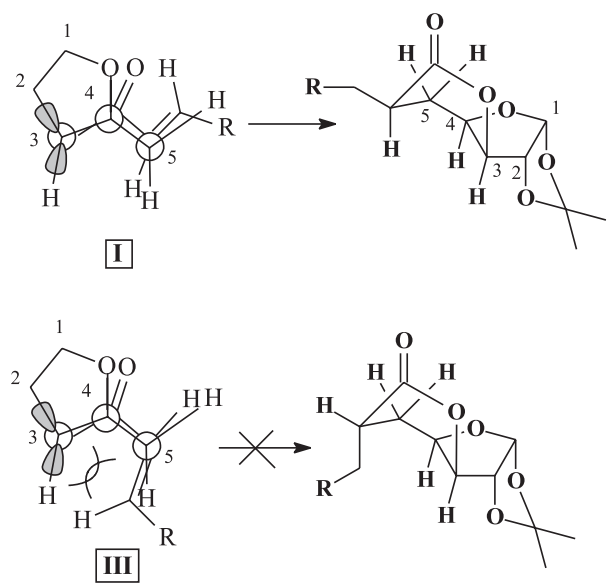

NOESY experiments allowed the establishment of the configuration of the newly formed stereocenters as $S$ for 9 and 10 (correlation between $\mathrm{H}-3$ and $\mathrm{H}-6$ ) and $R$ for $\mathbf{1 1}$ and 12 (correlation between $\mathrm{H}-4$ and H-7), as shown in Figure 3.

The stereoselectivity observed in these carbocyclization reactions was rationalized as being the result of the conformational trends of the radical esters in the respective transition states. The conformers of the radical esters in the furanose ring form and the corresponding $\delta$-lactones that should result from their cyclization are shown in Figure 4. Conformer I is less sterically hindered than conformers II, III and IV and, in consequence, the corresponding transition state should be favoured. This explains the formation of $\delta$-lactones 9 and $\mathbf{1 0 .}$

Similar arguments were used to rationalize the stereoselectivity observed in the cyclization of the radical esters in the pyranose ring form (Figure 5).

In conclusion, we describe herein the synthesis of carbohydrate-based $\delta$-lactones with complete regio- and stereoselectivity. This is an example of the "off-template" induction by the carbohydrate moiety on the diastereofacial selection. The configuration of the newly
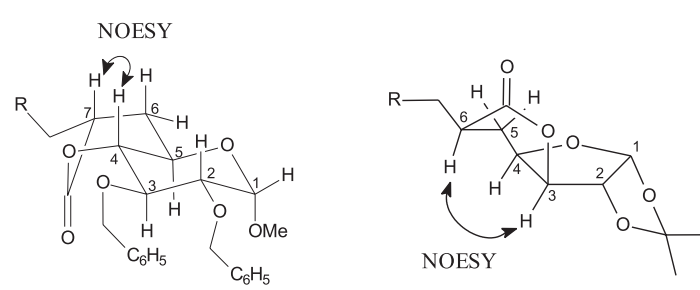

Figure 3. Correlation between $\mathrm{H}-4$ and $\mathrm{H}-7$ (pyranose ring forms) and $\mathrm{H}-3$ and $\mathrm{H}-6$ (furanose ring forms) observed by analysis of the NOESY contour maps.
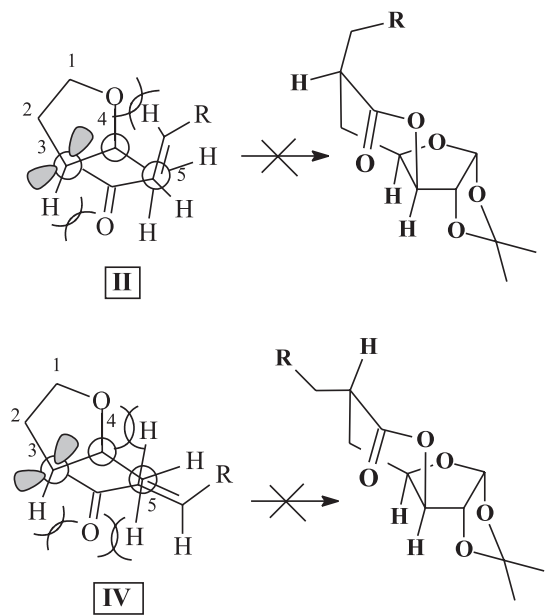

Figure 4. Conformers of the radical esters in the furanose ring forms and the corresponding $\delta$-lactones obtained from the cyclization of the least crowded conformer I. 


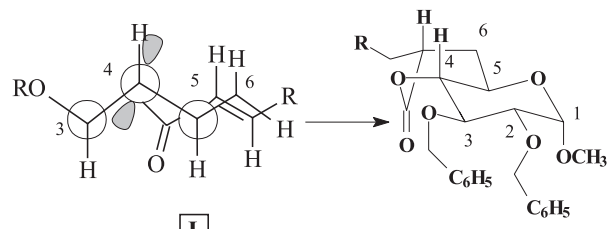

I

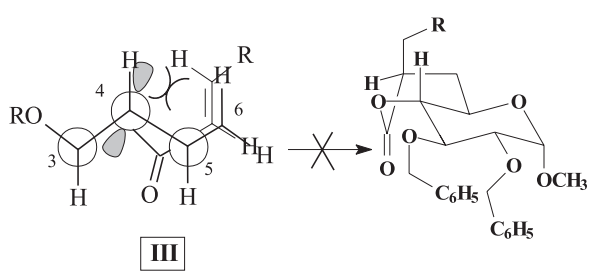

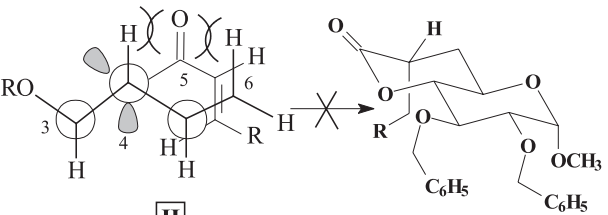

II

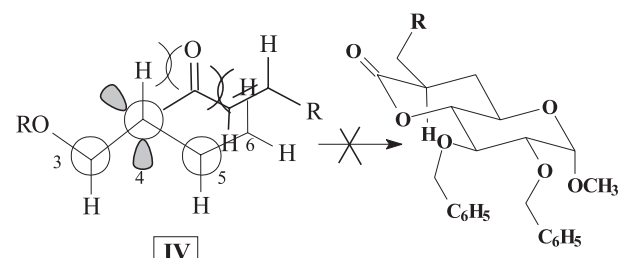

Figure 5. Conformers of the radical esters in the pyranose ring forms and the corresponding $\delta$-lactones obtained from the cyclization of the least crowded confomer I.

formed stereogenic center is dependent on the nature of the carbohydrate. Totally different stereochemical outcome is observed changing from the pyranose to the furanose ring forms.

\section{Experimental}

\section{General procedures}

All melting points were determined on a Kofler Sybron apparatus and are uncorrected. Optical rotations were determined at $25{ }^{\circ} \mathrm{C}$ with a Bellingham \& Stanley P20 Polarimeter. The NMR spectra were measured in deuteriochloroform or pyridine with TMS as the internal standard with a Bruker Avance DRX 400 or a Bruker Avance 200 instruments. Chemical shifts are given in $\delta$ scale and $J$ values are given in Hz. Column chromatography was performed with silica gel 60, 70-230 mesh (Merck).

General procedure for the synthesis of the radical precursors

To a 0.05 mol L-1 solution of compound $7^{10}$ or $\mathbf{8}^{22}$ in methylene chloride was added 4-dimethylaminopyridine (0.15 equivalents), carboxylic acid (cinnamic or crotonic acid) (1.5 equivalents) and $N, N^{\prime}$-dicyclohexylcarbodiimide (1.5 equivalents). The solution was allowed to stand at room temperature for $4 \mathrm{~h}$. The dicyclohexylurea which precipitates was removed by filtration. The filtrate was concentrated to give crude ester which was submitted to chromatography (hexane/EtOAc 8.5:1.5).

3-O-Cinnamoyl-5-deoxy-5-iodo-1,2-O-isopropylidene$\alpha$-D-xylofuranose (1). Following the general procedure, compound $7^{10}(0.50 \mathrm{~g}, 1.7 \mathrm{mmol})$ was treated with 4 dimethylaminopyridine, cinnamic acid and $N, N^{\prime}$ - dicyclohexylcarbodiimide. The oily residue was purified by column chromatography to give $\mathbf{1}(0.56 \mathrm{~g}, 79 \%)$ as a white solid: $\mathrm{mp} 115-116.8^{\circ} \mathrm{C}$; $[\alpha]_{\mathrm{D}}{ }^{25}-58\left(\mathrm{c} 2.0, \mathrm{CHCl}_{3}\right)$; $\delta_{\mathrm{H}}\left(200 \mathrm{MHz}, \mathrm{CDCl}_{3}\right) 7.74\left(1 \mathrm{H}, \mathrm{d}, J_{7,6} 16,7-\mathrm{H}\right) 7.56-7.40$ $(5 \mathrm{H}, \mathrm{m}, \mathrm{Ph}), 6.44\left(1 \mathrm{H}, \mathrm{d}, J_{6,7} 16,6-\mathrm{H}\right), 6.0\left(1 \mathrm{H}, \mathrm{d}, J_{1,2} 3.6\right.$, $1-\mathrm{H}), 5.46\left(1 \mathrm{H}, \mathrm{d}, J_{3,4} 3.2,3-\mathrm{H}\right), 4.64\left(1 \mathrm{H}, \mathrm{d}, J_{2,1} 3.6,2-\mathrm{H}\right)$, $4.61\left(1 \mathrm{H}\right.$, ddd, $\left.J_{4,3} 3.2, J_{4,5}=J_{4,5}, 6.0,4-\mathrm{H}\right), 3.31-3.27(2 \mathrm{H}$, $\mathrm{m}, 5-\mathrm{H}$ and 5 '-H), $1.56\left(3 \mathrm{H}, \mathrm{s}, \mathrm{CH}_{3}\right), 1.33\left(3 \mathrm{H}, \mathrm{s}, \mathrm{CH}_{3}\right) ; \delta_{\mathrm{C}}$ $\left(50 \mathrm{MHz}, \mathrm{CDCl}_{3}\right) 165.47(\mathrm{C}=\mathrm{O}), 146.45(7-\mathrm{C}), 133.91$ (C ipso), 130.76-128.24 (Ph), 116.54 (6-C), 112.41 $\left(\mathrm{C}\left(\mathrm{CH}_{3}\right)_{2}\right), 105.24$ (1-C), 83.46 (2-C), 79.75 (4-C), 76.24 (3-C), 26.74, 26.22 ( $2 \mathrm{x} \mathrm{CH}_{3}$ ), -2.43 (5-C) (Found: C, 47.71; $\mathrm{H}, 4.37$; Anal. Calc. for $\mathrm{C}_{17} \mathrm{H}_{19} \mathrm{IO}_{5}: \mathrm{C}, 47.44 ; \mathrm{H}, 4.42 \%$ ).

3-O-Crotonyl-5-deoxy-5-iodo-1,2-O-isopropylidene- $\alpha$ $D$-xylofuranose (2). Following the general procedure, compound $7^{10}(0.50 \mathrm{~g}, 1.7 \mathrm{mmol})$ was treated with 4dimethylaminopyridine, crotonic acid and $N, N^{\prime}-$ dicyclohexylcarbodiimide. The oily residue was purified by column chromatography to give $2(0.47 \mathrm{~g}, 77 \%)$ as a white solid: $\mathrm{mp} 65.4-65.7^{\circ} \mathrm{C} ;[\alpha]_{\mathrm{D}}^{25}-65.3\left(c 2.0, \mathrm{CHCl}_{3}\right)$; $\delta_{\mathrm{H}}\left(200 \mathrm{MHz}, \mathrm{CDCl}_{3}\right) 7.06\left(1 \mathrm{H}, \mathrm{dq}, J_{7.8} 6.9, J_{7.6} 15.5,7-\mathrm{H}\right)$, $5.95\left(1 \mathrm{H}, \mathrm{d}, J_{1,2} 3.6,1-\mathrm{H}\right), 5.87\left(1 \mathrm{H}, \mathrm{dd}, J_{6,8} 1.6, J_{6,7} 15.5,6-\right.$ $\mathrm{H}), 5.37\left(1 \mathrm{H}, \mathrm{d}, J_{3,4} 2.8,3-\mathrm{H}\right), 4.61-4.52(2 \mathrm{H}, \mathrm{m}, 2-\mathrm{H}$ and $4-$ H), $4.57\left(1 \mathrm{H}, \mathrm{d}, J_{2,1} 3.6,2-\mathrm{H}\right), 3.27\left(1 \mathrm{H}, \mathrm{dd}, J_{5,4} 6.6, J_{5,5}, 9.7\right.$, 5-H), 3.19 (1 H, dd, $\left.J_{5,4} 8.3, J_{5}, 59.7,5^{\prime}-\mathrm{H}\right), 1.92(3 \mathrm{H}$, dd, $\left.J_{8,6} 1.6, J_{8,7} 6.9,3 \times 8-\mathrm{H}\right), 1.54\left(3 \mathrm{H}, \mathrm{s}, \mathrm{CH}_{3}\right), 1.31(3 \mathrm{H}, \mathrm{s}$, $\left.\mathrm{CH}_{3}\right) ; \delta_{\mathrm{C}}\left(50 \mathrm{MHz}, \mathrm{CDCl}_{3}\right) 164.89(\mathrm{C}=\mathrm{O}), 146.74(7-\mathrm{C})$, $121.46(6-\mathrm{C}), 112.33\left(\mathrm{C}\left(\mathrm{CH}_{3}\right)_{2}\right), 105.19(1-\mathrm{C}), 83.40(2-\mathrm{C})$, 79.72 (4-C), 75.89 (3-C), 26.73, $26.19\left(2 \mathrm{x} \mathrm{CH}_{3}\right.$ ), 18.11 (8C), -2.41 (5-C); (Found: C, 39.12; H, 4.36; Anal. Calc. for $\left.\mathrm{C}_{12} \mathrm{H}_{17} \mathrm{IO}_{5}: \mathrm{C}, 39.13 ; \mathrm{H}, 4.62 \%\right)$.

Methyl 2,3-di-O-benzyl-4-O-cinnamoyl-6-deoxy-6iodo- $\alpha$-D-glucopyranoside (3). Following the general procedure, compound $\mathbf{8}^{22}(0.52 \mathrm{~g}, 1.1 \mathrm{mmol})$ was treated with 4-dimethylaminopyridine, cinnamic acid and $N, N^{\prime}$ - 
dicyclohexylcarbodiimide. The oily residue was purified by column chromatography to give $3(0.37 \mathrm{~g}, 56 \%)$ as a colorless oil: $[\alpha]_{\mathrm{D}}^{25}-38\left(c 2.0, \mathrm{CHCl}_{3}\right) ; \delta_{\mathrm{H}}(200 \mathrm{MHz}$, $\left.\mathrm{CDCl}_{3}\right) 7.66\left(1 \mathrm{H}, \mathrm{d}, J_{8,7} 16,8-\mathrm{H}\right), 7.54-7.15(15 \mathrm{H}, \mathrm{m}, \mathrm{Ph})$, 6.29 (1 H, d, $\left.J_{7,8} 16,7-\mathrm{H}\right), 4.92\left(1 \mathrm{H}, \mathrm{t}, J_{4,3}=\mathrm{J}_{4,5} 9.4,4-\mathrm{H}\right)$, 4.89-4.62 (5 H, m, $4 \mathrm{CH}_{2} \mathrm{Ph}$ and 1-H), $3.99\left(1 \mathrm{H}, \mathrm{t}, J_{3,4}=J_{3,2}\right.$ 9.4, 3-H), $3.77\left(1 \mathrm{H}, \mathrm{td}, J_{5,6}, 2.6, J_{5,6}=J_{5,4} 9.4,5-\mathrm{H}\right), 3.61(1 \mathrm{H}$, dd, $\left.J_{2,1} 3.6, J_{2,3} 9.4,2-\mathrm{H}\right), 3.49\left(3 \mathrm{H}, \mathrm{s}, \mathrm{OCH}_{3}\right), 3.27(1 \mathrm{H}, \mathrm{dd}$, $\left.J_{6,5} 2.6, J_{6,6} 10.8,6^{\prime}-\mathrm{H}\right), 3.09$ (1 H, dd, $J_{6,5} 9.4, J_{6,6}, 10.8,6-$ $\mathrm{H}) ; \delta_{\mathrm{C}}\left(50 \mathrm{MHz} \mathrm{CDCl}_{3}\right) 165.7(\mathrm{C}=\mathrm{O}), 146.0(8-\mathrm{C}), 138.1$ (one of C ipso), 137.8 (other of $\mathrm{C}$ ipso), 134.0 (other of C ipso), 130.6-127.5 (Ph), 117.0 (7-C), 98.2 (1-C), 79.6 (2C), 78.4 (3-C), 75.3, $73.6\left(2 \times \mathrm{PhCH}_{2} \mathrm{O}\right), 71.9$ (4-C), 69.5 (5-C), $55.8\left(\mathrm{OCH}_{3}\right), 4.5$ (6-C); (Found: C, 58.36; H, 5.14; Anal. Calc. for $\mathrm{C}_{30} \mathrm{H}_{31} \mathrm{IO}_{6}: \mathrm{C}, 58.63 ; \mathrm{H}, 5.05 \%$ ).

Methyl 2,3-di-O-benzyl-4-O-crotonyl-6-deoxy-6-iodo$\alpha$-D-glucopyranoside (4). Following the general procedure, compound $\mathbf{8}^{22}(0.39 \mathrm{~g}, 0.8 \mathrm{mmol})$ was treated with 4-dimethylaminopyridine, crotonic acid and $N, N^{\prime}-$ dicyclohexylcarbodiimide. The oily residue was purified by column chromatography to give $4(0.25 \mathrm{~g}, 56 \%)$ as a colorless oil: $[\alpha]_{\mathrm{D}}{ }^{25}-20.2\left(c 1.6, \mathrm{CHCl}_{3}\right) ; \delta_{\mathrm{H}}(200 \mathrm{MHz}$, $\left.\mathrm{CDCl}_{3}\right)$ 7.36-7.21 (10 H, m, Ph), $6.98\left(1 \mathrm{H}, \mathrm{dq}, J_{8,9} 6.9, J_{8,7}\right.$ $15.5,8-\mathrm{H}), 5.76\left(1 \mathrm{H}, \mathrm{dd}, J_{7,9} 1.7, J_{7,8} 15.5,7-\mathrm{H}\right), 4.89-4.60$ (5 H, m, 4 x CH $2 \mathrm{Ph}, 4-\mathrm{H}), 4.61$ (1 H, d, J $3.6,1-\mathrm{H}), 3.93$ (1 $\left.\mathrm{H}, \mathrm{t}, J_{3,2}=J_{3,4} 9.6,3-\mathrm{H}\right), 3.73\left(1 \mathrm{H}, \mathrm{td}, J_{5,6} 2.4, J_{5,4}=J_{5,6} 9.6\right.$, 5-H), $3.57\left(1 \mathrm{H}, \mathrm{dd}, J_{2,1} 3.6, J_{2,3} 9.6,2-\mathrm{H}\right), 3.47\left(3 \mathrm{H}, \mathrm{s}, \mathrm{OCH}_{3}\right)$, $3.23\left(1 \mathrm{H}, \mathrm{dd}, J_{6,5} 2.4, J_{6,6} 10.8,6^{\prime}-\mathrm{H}\right), 3.05$ ( $1 \mathrm{H}, J_{6,5} 9.6, J_{6,6}$ ' $10.8,6-\mathrm{H}), 1.88$ (3 H, dd, $\left.J_{9,7} 1.6, J_{9,8} 6.9,3 \times 9-\mathrm{H}\right) ; \delta_{\mathrm{C}}(50$ $\mathrm{MHz} \mathrm{CDCl}_{3}$ ) 165.20 (C=O), 146.29 (8-C), 138.25, 137.86 (2 x C ipso), 128.47-127.49 (Ph); 121.89 (7-C), 98.18 (1C), 79.49 (2-C), 78.57 (3-C), 75.36, 73.55 ( $\left.2 \times \mathrm{PhCH}_{2} \mathrm{O}\right)$, 73.37 (4-C), 69.56 (5-C), $55.72\left(\mathrm{OCH}_{3}\right), 18.08$ (C-9), 4.48 (6-C); (Found: C, 54.87; H, 5.22; Anal. Calc. for $\mathrm{C}_{25} \mathrm{H}_{29} \mathrm{IO}_{6}$ : C, $54.35 ; \mathrm{H}, 5.25 \%)$.

\section{General procedure for free radical cyclization}

To a stirred, boiling solution of radical precursor in nitrogen-saturated benzene $\left(0.01 \mathrm{~mol} \mathrm{~L}^{-1}\right)$ was added a solution of $\mathrm{Bu}_{3} \mathrm{SnH}$ (1.2 equivalents) and AIBN (cat.) in nitrogen-saturated benzene $\left(0.2 \mathrm{~mol} . \mathrm{L}^{-1}\right)$ via an addition funnel during $3 \mathrm{~h}$. The reaction mixture was heated under reflux in nitrogen atmosphere for a further $1 \mathrm{~h}$. After solvent removal the residue was dissolved in acetonitrile $(50 \mathrm{~mL})$. The solution was extracted three times with hexane (30 $\mathrm{mL}$ ) to remove tin compounds. After evaporation of the acetonitrile the residue was chromatographed (hexane/ EtOAc 8:2).

Free radical cyclization of radical precursor 1 . Starting from 1 (0.47 g, $1.1 \mathrm{mmol})$ and following the general procedure, the $\delta$-lactone $9(0.10 \mathrm{~g}, 30 \%)$ and the uncyclized product $13(0.14 \mathrm{~g}, 42 \%)$ were isolated. The $\delta$-lactone 9 was obtained as a white solid; $\mathrm{mp} 138.5-139.7^{\circ} \mathrm{C} ;[\alpha]_{\mathrm{D}}^{25}+$ $218\left(c\right.$ 0.37, $\left.\mathrm{CHCl}_{3}\right) ; \delta_{\mathrm{H}}\left(400 \mathrm{MHz}, \mathrm{CDCl}_{3}\right) 7.33-7.18(5 \mathrm{H}$, m, Ph), 5.94 (1 H, d, $\left.J_{1,2} 3.6,1-\mathrm{H}\right), 4.75$ (1 H, d, $\left.J_{2,1} 3.6,2-\mathrm{H}\right)$, 4.60-4.56 (1 H, m, 4-H), 4.55 (1 H, d, J 3.4 2.8, 3-H), 3.35 (1 $\left.\mathrm{H}, \mathrm{dd}, J_{7,6} 4.4, J_{7,7}, 14.0,7-\mathrm{H}\right) ; 2.61\left(1 \mathrm{H}, \mathrm{dd}, J_{7,6} 9.4, J_{7,7}\right.$ 14.0, 7'-H), 2.54-2.46 (1 H, m, 6-H), 2.36 (1 H, ddd, $J_{5,4} 6.4$, $\left.J_{5,6} 8.2, J_{5,5}, 14.2,5-\mathrm{H}\right), 1.66\left(1 \mathrm{H}, \mathrm{ddd}, J_{5^{\prime}, 4} 3.8, J_{5^{\prime}, 6} 12.8, J_{5^{\prime}, 5}\right.$ 14.2, 5'-H), 1.46 (3 H, s, $\left.\mathrm{CH}_{3}\right), 1.32\left(3 \mathrm{H}, \mathrm{s}, \mathrm{CH}_{3}\right) ; \delta_{\mathrm{C}}(100$ $\mathrm{MHz}, \mathrm{CDCl}_{3}$ ) $172.92(\mathrm{C}=\mathrm{O}), 138.45$ (C ipso), 129.11, 128.60, $126.66(\mathrm{Ph}), 112.09\left(\mathrm{C}\left(\mathrm{CH}_{3}\right)_{2}\right), 104.86(1-\mathrm{C}), 83.21$ (2-C), 81.70 (3-C), 73.65 (4-C), 39.35 (6-C), 36.25 (7-C), 28.10 (5-C), 26.6, 26.2 (2 x $\mathrm{CH}_{3}$ ); (Found: C, 67.32; H, 6.64; Anal. Calc. for $\mathrm{C}_{17} \mathrm{H}_{20} \mathrm{O}_{5}: \mathrm{C}, 67.09 ; \mathrm{H}, 6.62 \%$ ).

The uncyclized product $\mathbf{1 3}$ was obtained as a white solid; mp 67.9-69.3 ${ }^{\circ} \mathrm{C}$, $\operatorname{lit}^{23} 73-75^{\circ} \mathrm{C}$; $[\alpha]_{\mathrm{D}}{ }^{25}+56$ (c 1.14, $\left.\mathrm{CHCl}_{3}\right), \mathrm{lit}^{23}+44(c 4.72$, benzene $) ; \delta_{\mathrm{H}}\left(200 \mathrm{MHz}, \mathrm{CDCl}_{3}\right)$ $7.73\left(1 \mathrm{H}, \mathrm{d}, J_{7,6} 16.0,7-\mathrm{H}\right), 7.56-7.38(5 \mathrm{H}, \mathrm{m}, \mathrm{Ph}), 6.46$ (1 H, d, $\left.J_{6,7} 16.0,6-\mathrm{H}\right), 5.95\left(1 \mathrm{H}, \mathrm{d}, J_{1,2} 3.8,1-\mathrm{H}\right), 5.25(1 \mathrm{H}$, $\left.\mathrm{d}, J_{3,4} 2.8,3-\mathrm{H}\right), 4.61\left(1 \mathrm{H}, \mathrm{d}, J_{2,1} 3.8,2-\mathrm{H}\right), 4.49$ (1 H, qd, $J_{4,3}$ 2.8, $\left.J_{4,5} 6.4,4-\mathrm{H}\right), 1.54\left(3 \mathrm{H}, \mathrm{s}, \mathrm{CH}_{3}\right), 1.32\left(3 \mathrm{H}, \mathrm{s}, \mathrm{CH}_{3}\right), 1.29$ $\left(3 \mathrm{H}, \mathrm{d}, J_{5,4} 6.4,3 \times 5-\mathrm{H}\right) ; \delta_{\mathrm{C}}\left(50 \mathrm{MHz}, \mathrm{CDCl}_{3}\right) 165.66(\mathrm{C}=\mathrm{O})$, 145.86 (7-C), 133.94 (C ipso), 130.50, 128.83, 128.07 (Ph), 116.89 (6-C), $111.56\left(\mathrm{C}\left(\mathrm{CH}_{3}\right)_{2}\right), 104.41(1-\mathrm{C}), 83.85$ (2-C), 77.46 (3-C), 75.13 (4-C), 26.47, 26.04 (2 x $\left.\mathrm{CH}_{3}\right), 13.09$ (5-C).

Free radical cyclization of radical precursor 2 . Starting from $2(0.50 \mathrm{~g}, 1.4 \mathrm{mmol})$ and following the general procedure, the $\delta$-lactone $\mathbf{1 0}(0.05 \mathrm{~g}, 15 \%)$ and the uncyclized product $14(0.22 \mathrm{~g}, 66 \%)$ were isolated. The $\delta$ lactone 10 was obtained as a white solid; $\mathrm{mp} 91.5-93.4^{\circ} \mathrm{C}$; $[\alpha]_{\mathrm{D}}^{25}+103\left(c 1.3, \mathrm{CHCl}_{3}\right) ; \delta_{\mathrm{H}}\left(200 \mathrm{MHz}, \mathrm{CDCl}_{3}\right) 5.93(1$ $\left.\mathrm{H}, \mathrm{d}, J_{1,2} 3.8,1-\mathrm{H}\right), 4.75\left(1 \mathrm{H}, \mathrm{d}, J_{2,1} 3.8,2-\mathrm{H}\right), 4.72-4.66(1 \mathrm{H}$, m, 4-H), 4.57 (1 H, d, $\left.J_{3,4} 2.8,3-\mathrm{H}\right), 2.52$ (1 H, ddd, $J_{5,6} 6.4$, $\left.J_{5,4} 8.9, \mathrm{~J}_{5,5} 14.1,5^{\prime}-\mathrm{H}\right), 2.18\left(1 \mathrm{H}, \mathrm{dq}, J_{6,5}=J_{6,7}=J_{6,7} 6.4\right.$, $\left.J_{6,5}, 12.9,6-\mathrm{H}\right), 1.89\left(1 \mathrm{H}, \mathrm{tdd}, J_{7,6} 6.4, J_{7,8(\mathrm{CH} 3)} 7.6, J_{7,7}, 14,1\right.$, 7-H), 1.73-1.38 (5 H, m, 5-H, 7'- $\mathrm{H}$ and $\left.\mathrm{CH}_{3}\right), 1.33$ (3 H, s, $\left.\mathrm{CH}_{3}\right), 1.00\left(3 \mathrm{H}, \mathrm{t}, J_{8,7}=J_{8,7}, 7.6 \mathrm{~Hz}, 8-\mathrm{H}\left(\mathrm{CH}_{3}\right) ; \delta_{\mathrm{C}}(50 \mathrm{MHz}\right.$, $\left.\mathrm{CDCl}_{3}\right) 173.37(\mathrm{C}=\mathrm{O}), 111.98\left(\mathrm{C}\left(\mathrm{CH}_{3}\right)_{2}\right), 104.77(1-\mathrm{C})$, 83.06 (2-C), 81.39 (3-C), 72.06 (4-C), 38.73 (6-C), 28.44 (5-C), 26.54, 26.10 (2 x $\mathrm{CH}_{3}$ ), 22.88 (7-C), 11.24 (8-C); (Found: C, 59.62, H 7.35; Calc. for $\mathrm{C}_{12} \mathrm{H}_{18} \mathrm{O}_{5}$ : C, 59.49, H, $7.49 \%$ ).

The uncyclized product $\mathbf{1 4}$ was obtained as an oil; $[\alpha]_{\mathrm{D}}+31.9(c 2.8$, benzene $)$, lit $^{23}+31.0(c 2.8$, benzene $) ; \delta_{\mathrm{H}}$ $\left(200 \mathrm{MHz} \mathrm{CDCl}_{3}\right) 7.04\left(1 \mathrm{H}, \mathrm{dq}, J_{7,8} 7.0, J_{7,6} 15.5,7-\mathrm{H}\right)$, 5.90 (1 H, d, $\left.J_{1,2} 3.8,1-\mathrm{H}\right), 5.91-5.83(1 \mathrm{H}, \mathrm{m}, 6-\mathrm{H}), 5.16$ (1 $\left.\mathrm{H}, J_{3,4} 2.8,3-\mathrm{H}\right), 4.55\left(1 \mathrm{H}, \mathrm{d}, J_{2,1} 3.8,2-\mathrm{H}\right), 4.47$ (1 H, qd, $J_{4,3}$ $\left.2.8, J_{4,5} 6.4,4-\mathrm{H}\right), 1.90\left(3 \mathrm{H}, \mathrm{dd}, J_{8,6} 1.6, \mathrm{~J}_{8,7} 7.0,3 \times 8-\mathrm{H}\right)$, $1.52\left(3 \mathrm{H}, \mathrm{s}, \mathrm{CH}_{3}\right), 1.31\left(3 \mathrm{H}, \mathrm{s}, \mathrm{CH}_{3}\right), 1.24\left[3 \mathrm{H}, \mathrm{d}, J_{5,4} 6.4,5-\right.$ 
$\left.\mathrm{H}\left(\mathrm{CH}_{3}\right)\right] ; \delta_{\mathrm{C}}\left(50 \mathrm{MHz}, \mathrm{CDCl}_{3}\right) 165.17(\mathrm{C}=\mathrm{O}), 146.09$ (7-C), $\left.121.72(6-\mathrm{C}), 111.54\left(\mathrm{C}_{\left(\mathrm{CH}_{3}\right.}\right)_{2}\right), 104.40$ (1-C), 83.86 (2-C), 77.14 (3-C), 75.14 (4-C), 26.49, $26.05\left(2 \times \mathrm{CH}_{3}\right), 17.96$ (8C), 13.06 (5-C).

Free radical cyclization of radical precursor 3 . Starting from $3(0.40 \mathrm{~g}, 0.65 \mathrm{mmol})$ and following the general procedure, $\delta$-lactone $\mathbf{1 1}(0.07 \mathrm{~g}, 22 \%)$ and the uncyclized product $15(0.17 \mathrm{~g}, 53 \%)$ were isolated. The $\delta$-lactone $\mathbf{1 1}$ was obtained as a white solid; $\mathrm{mp}$ 131.2$132 .{ }^{\circ} \mathrm{C} ;[\alpha]_{\mathrm{D}}^{25}-30\left(c 0.8, \mathrm{CHCl}_{3}\right) ; \delta_{\mathrm{H}}(400 \mathrm{MHz}, \mathrm{Py})$ 7.56-7.24 $(15 \mathrm{H}, \mathrm{m}, \mathrm{Ph}), 5.07\left(1 \mathrm{H}, \mathrm{d}, J_{\text {gem }} 11.6\right.$, one of $\left.\mathrm{PhCH}_{2}\right), 5.02\left(1 \mathrm{H}, \mathrm{d}, J_{\text {gem }} 11.6\right.$, one of $\left.\mathrm{PhCH}_{2}\right), 4.95(1 \mathrm{H}, \mathrm{d}$, $\left.J_{1,2} 3.6,1-\mathrm{H}\right), 4.84\left(1 \mathrm{H}, \mathrm{d}, J_{\text {gem }} 12.0\right.$, one of $\left.\mathrm{PhCH}_{2}\right), 4.79$ (1 $\mathrm{H}, \mathrm{d}, J_{\mathrm{gem}} 12.0$, one of $\left.\mathrm{PhCH}_{2}\right), 4.42\left(1 \mathrm{H}, \mathrm{t}, J_{4,3}=J_{4,5} 9.2,4-\right.$ $\mathrm{H}), 4.24\left(1 \mathrm{H}, \mathrm{t}, J_{3,4}=J_{3,2} 9.2,3-\mathrm{H}\right), 3.95\left(1 \mathrm{H}, \mathrm{q}, J_{5,4}=J_{5,6}=\right.$ $\left.J_{5,6} 9.2,5-\mathrm{H}\right), 3.76\left(1 \mathrm{H}, \mathrm{dd}, J_{2,1} 3.6, J_{2,3} 9.2,2-\mathrm{H}\right), 3.46(1 \mathrm{H}$, dd, $\left.J_{8,7} 4.8, J_{8,8}, 13.8,8-\mathrm{H}\right), 3.41-3.33\left(4 \mathrm{H}, \mathrm{m}, 7-\mathrm{H}\right.$ and $\left.\mathrm{OCH}_{3}\right)$, $2.82\left(1 \mathrm{H}, \mathrm{dd}, J_{8^{\prime}, 7} 9.2, J_{8^{\prime}, 8} 13.8,8^{\prime}-\mathrm{H}\right), 2.02-1.88(2 \mathrm{H}, \mathrm{m}, 6-$ $\mathrm{H}$ and 6 '-H); $\delta_{\mathrm{C}}(100 \mathrm{MHz}, \mathrm{Py}) 173.72(\mathrm{C}=\mathrm{O}), 139.51$, 139.46, 139.14 (3 x C ipso), 129.60-126.89 (Ph), 98.89 (1C), 79.83 (2-C), 79.15 (4-C), 79.04 (3-C), 75.20, 73.13 ( 2 x $\left.\mathrm{PHCH}_{2} \mathrm{O}\right), 64.07$ (5-C), $55.32\left(\mathrm{OCH}_{3}\right), 38.62$ (7-C), 37.28 (8-C), 29.63 (6-C).

The uncyclized product 15 was obtained as an oil; $[\alpha]_{\mathrm{D}}^{25}$ $+12.5\left(c 2.0, \mathrm{CHCl}_{3}\right) ; \delta_{\mathrm{H}}\left(400 \mathrm{MHz}, \mathrm{CDCl}_{3}\right) 7.65\left(1 \mathrm{H}, \mathrm{d}, J_{8,7}\right.$ $16.0,8-\mathrm{H}), 7.52-7.15(15 \mathrm{H}, \mathrm{m}, \mathrm{Ph}), 6.31\left(1 \mathrm{H}, \mathrm{d}, J_{7.8} 16.0,7-\right.$ $\mathrm{H}), 4.88\left(1 \mathrm{H}, \mathrm{t}, J_{4.3}=J_{4.5} 9.6,4-\mathrm{H}\right), 4.86\left(1 \mathrm{H}, \mathrm{d}, J_{\text {gem }} 11.6\right.$, one of $\left.\mathrm{PhCH}_{2}\right), 4.81\left(1 \mathrm{H}, \mathrm{d}, J_{\text {gem }} 12.4\right.$, one of $\left.\mathrm{PhCH}_{2}\right), 4.69-4.66$ $\left(2 \mathrm{H}, \mathrm{m}, 2 \mathrm{x} \mathrm{PhCH}_{2}\right), 4.56\left(1 \mathrm{H}, \mathrm{d}, J_{1,2} 3.6,1-\mathrm{H}\right), 3.95(1 \mathrm{H}, \mathrm{t}$, $\left.J_{3,4}=J_{3,2} 9.6,3-\mathrm{H}\right), 3.82\left(1 \mathrm{H}, \mathrm{qd}, J_{5,6(\mathrm{CH} 3)} 6.4, J_{5,4} 9.6,5-\mathrm{H}\right)$, $3.61\left(1 \mathrm{H}, \mathrm{dd}, J_{2,1} 3.6, J_{2,3} 9.6,2-\mathrm{H}\right), 3.40\left(3 \mathrm{H}, \mathrm{s}, \mathrm{OCH}_{3}\right), 1.15$ $\left[3 \mathrm{H}, \mathrm{d}, J_{6(\mathrm{CH} 3), 5} 6.4,6-\mathrm{H}\left(\mathrm{CH}_{3}\right)\right] ; \delta_{\mathrm{C}}\left(100 \mathrm{MHz}, \mathrm{CDCl}_{3}\right) 165.85$ $(\mathrm{C}=\mathrm{O}), 145.26$ (8-C), 138.57, 138.15, 134.37 (3 x C ipso), 128.87-127.38 (Ph), 117.79 (7-C), 98.23 (1-C), 80.04 (2-C), 79.01 (3-C), 75.28 (4-C), 75.23, $75.18\left(2 \mathrm{x} \mathrm{PhCH}_{2} \mathrm{O}\right), 73.44$ (5-C), $55.30\left(\mathrm{OCH}_{3}\right), 17.39$ (6-C).

Free radical cyclization of radical precursor 4 . Starting from $4(0.47 \mathrm{~g}, 0.85 \mathrm{mmol})$ and following the general procedure, $\delta$-lactone $\mathbf{1 2}(0.004 \mathrm{~g}, 1 \%)$ and the uncyclized product $16(0.25 \mathrm{~g}, 70 \%)$ were isolated.

The $\delta$-lactone 12 was obtained as a white solid; $\delta_{\mathrm{H}}(400$ $\left.\mathrm{MHz}, \mathrm{CDCl}_{3}\right) 7.30-7.39(10 \mathrm{H}, \mathrm{m}, \mathrm{Ph}), 4.93\left(1 \mathrm{H}, \mathrm{d}, J_{\text {gem }} 10.8\right.$, one of $\left.\mathrm{PhCH}_{2}\right), 4.85\left(1 \mathrm{H}, \mathrm{d}, J_{\text {gem }} 10.8\right.$, one of $\left.\mathrm{PhCH}_{2}\right)$, 4.83 (1 $\mathrm{H}, \mathrm{d}, J_{\text {gem }} 12.0$, one of $\left.\mathrm{PhCH}_{2}\right), 4.66\left(1 \mathrm{H}, \mathrm{d}, J_{\text {gem }} 12.0\right.$, one of $\left.\mathrm{PhCH}_{2}\right), 4.55\left(1 \mathrm{H}, \mathrm{d}, J_{1,2} 3.6,1-\mathrm{H}\right), 4.04\left(1 \mathrm{H}, \mathrm{t}, J_{4,3}=J_{4,5} 9.2\right.$, 4-H), $4.01\left(1 \mathrm{H}, \mathrm{t}, J_{3,4}=J_{3,2} 9.2,3-\mathrm{H}\right), 3.86\left(1 \mathrm{H}, \mathrm{q}, J_{5,4}=J_{5,6}=\right.$ $\left.J_{5,6} 9.2,5-\mathrm{H}\right), 3.51\left(1 \mathrm{H}, \mathrm{dd}, J_{2,1} 3.6, J_{2,3} 9.2,2-\mathrm{H}\right), 3.39(3 \mathrm{H}$, $\left.\mathrm{s}, \mathrm{OCH}_{3}\right), 2.61-2.52(1 \mathrm{H}, \mathrm{m}, 7-\mathrm{H}), 2.05\left(1 \mathrm{H}\right.$, ddd, $J_{6,5} 9.2, J_{6,7}$ $\left.10.4, J_{6,6}, 13.5,6-\mathrm{H}\right), 1.96-1.79\left(2 \mathrm{H}, \mathrm{m}, 6^{\prime}-\mathrm{H}\right.$ and $\left.8-\mathrm{H}\right), 1.48$ (1 H, double quintet, $\left.J_{8^{\prime}, 7}=J_{8^{\prime}, 9 \text { (CH3) }} 7.6, J_{8^{\prime}, 8} 14.3,8^{\prime}-\mathrm{H}\right), 0.97$ $\left[3 \mathrm{H}, \mathrm{t}, J_{9(\mathrm{CH} 3), 8}=J_{9(\mathrm{CH} 3), 8}, 7.6,9-\mathrm{H}\left(\mathrm{CH}_{3}\right)\right] ; \delta_{\mathrm{C}}\left(100 \mathrm{MHz}, \mathrm{CDCl}_{3}\right)$
$173.63(\mathrm{C}=\mathrm{O}), 138.43,138.01$ ( 2 x C ipso), 128.45-127.65 ( $\mathrm{Ph}), 98.91$ (1-C), 79.13 (4-C), 78.83 (3-C), 78.74 (2-C), 75.55, $73.75\left(2 \times \mathrm{PHCH}_{2} \mathrm{O}\right), 63.65(5-\mathrm{C}), 55.57\left(\mathrm{OCH}_{3}\right), 38.14$ (7C), 29.25 (6-C), 23.87 (8-C), 11.17 (9-C).

The uncyclized product $\mathbf{1 6}$ was obtained as an oil; $[\alpha]_{\mathrm{D}}^{25}+58.7\left(c 2.0, \mathrm{CHCl}_{3}\right) ; \delta_{\mathrm{H}}\left(200 \mathrm{MHz}, \mathrm{CDCl}_{3}\right) 7.36-$ $7.22(10 \mathrm{H}, \mathrm{m}, \mathrm{Ph}), 6.96\left(1 \mathrm{H}, \mathrm{dq}, J_{8.9(\mathrm{CH} 3)} 6.9, J_{8.7} 15.5,8-\mathrm{H}\right)$, $5.77\left(1 \mathrm{H}, \mathrm{dd}, J_{7,9(\mathrm{CH})} 1.7, J_{7,8} 15.5,7-\mathrm{H}\right), 4.86-4.61(5 \mathrm{H}, \mathrm{m}$, $4 \times \mathrm{PhCH}_{2}$ and 4-H), $4.54\left(1 \mathrm{H}, \mathrm{d}, J_{1,2} 3.6,1-\mathrm{H}\right), 3.90(1 \mathrm{H}, \mathrm{t}$, $\left.J_{3,4}=J_{3,2} 9.4,3-\mathrm{H}\right), 3.81-3.70(1 \mathrm{H}, \mathrm{m}, 5-\mathrm{H}), 3.57(1 \mathrm{H}, \mathrm{dd}$, $\left.J_{2,1} 3.6, J_{2,3} 9.4,2-\mathrm{H}\right), 3.38\left(3 \mathrm{H}, \mathrm{s}, \mathrm{OCH}_{3}\right), 1.87[3 \mathrm{H}, \mathrm{dd}$, $\left.J_{9(\mathrm{CH} 3), 7} 1.7, J_{9(\mathrm{CH} 3), 8} 6.9,9-\mathrm{H}\left(\mathrm{CH}_{3}\right)\right], 1.12\left[3 \mathrm{H}, \mathrm{d}, J_{6(\mathrm{CH} 3), 5} 6.2\right.$, 6- $\left.\mathrm{H}\left(\mathrm{CH}_{3}\right)\right] ; \delta_{\mathrm{C}}\left(50 \mathrm{MHz}, \mathrm{CDCl}_{3}\right) 165.35(\mathrm{C}=\mathrm{O}), 145.36(8-$ C), 138.49, $138.02(2 \times$ C ipso $), 128.37-127.35(\mathrm{Ph})$, 122.30 (7-C), 98.11 (1-C), 79.74 (2-C), 78.99 (3-C), 75.17, 73.40 ( $2 \times \mathrm{PhCH}_{2} \mathrm{O}$ ), 74.88 (4-C), 65.37 (5-C), 55.19 $\left(\mathrm{OCH}_{3}\right), 17.97$ [9-C $\left.\left(\mathrm{CH}_{3}\right)\right], 17.26\left[6-\mathrm{C}\left(\mathrm{CH}_{3}\right)\right]$.

\section{Acknowledgements}

We wish to thank Fundação de Amparo à Pesquisa do Estado de Minas Gerais (FAPEMIG) and Conselho Nacional de Desenvolvimento Científico e Tecnológico (CNPq) for financial support.

\section{References}

1. Jasperse, C. P.; Curran, D. P.; Fevig, T. L.; Chem. Rev. 1991, 91, 1237.

2. Mori, K.; Tetrahedron 1989, 45, 3233.

3. Koch, S. S. C.; Chamberline, A. R.; J. Org. Chem. 1993, 58, 2725 .

4. Sharma, G. V. M.; Vepachedu, S. R.; Tetrahedron Lett. 1990, 31,4931 .

5. Xi, Z.; Agback, P.; Sandströn, A.; Chattopadhyaya, J.; Tetrahedron 1991, 47, 9675

6. Velázquez, S.; Camarasa, M. J.; Tetrahedron Asym. 1994, 5, 2141.

7. Prado, M. A. F.; Alves, R. J.; Souza Filho, J. D.; Alves, R. B.; Pedrosa, M. T. C.; Prado, R. F.; Faraco, A. A. G.; J. Chem. Soc., Perkin Trans. 1. 2000, 1853.

8. Binatti, I.; Prado, M. A. F.; Alves, R. J.; Souza Filho, J. D.; J. Braz. Chem. Soc. 2002, 13, 570.

9. Faraco, A. A. G.; Prado, M. A. F.; Alves, R. J.; Souza Filho, J. D.; Alves, R. B.; Faraco, R. F. P.; Synth. Commun. 2003, 33, in press.

10. Levene, P. A.; Raymond, A. L.; J. Biol. Chem. 1933, 102, 317.

11. Bell, D. J.; Lorber, J.; J. Chem. Soc. 1940, 453.

12. Garegg, P. J.; Samuelson, B. J.; J. Chem. Soc., Perkin Trans. 1. 1980, 2866.

13. Hassner, A.; Alexanian, V.; Tetrahedron Lett. 1978, 46, 4475. 
14. Oki, M.; Nakanishi, H.; Bull. Chem. Soc. Jpn. 1970, 43, 2558.

15. Curran, D. P.; Chang, C. T.; J. Org. Chem. 1989, 54, 3140.

16. Baldwin, J. E.; Adlington, R. M.; Mitchell, M. B.; Robertson, J.; J. Chem. Soc., Chem. Commun. 1990, 1574.

17. Barth, F.; O-Yang, C.; Tetrahedron Lett. 1990, 31, 1121.

18. Ishizaki, M.; Ozaki, K.; Kanematsu, A.; Isoda, T.; Hoshino, O.; J. Org. Chem. 1993, 58, 3877.

19. Junggebauer, J.; Neumann, W. P.; Tetrahedron 1997, 53, 1301.
20. Bowman, W. R.; Mann, E.; Parr, J.; J. Chem. Soc., Perkin Trans. 1 2000, 2991.

21. Curran, D. P.; Porter, N. A.; Giese, B.; Stereochemistry of Radical Reactions, VHC: Weinheim, 1996, p. 280.

22. Köhn, A.; Schmidt, R. R.; Liebigs Ann. Chem. 1987, 1045.

23. Kawana, M.; Emoto, S.; Bull. Chem. Soc. Jpn. 1966, 39, 910.

Received: January 3, 2002

Published on the web: February 21, 2003 